\title{
3-D Electron Microscopy for Nano-Technology and the IC Industry
}

\author{
C. Kübel*, D. Hubert, W.F. Voorhout, M.T. Otten \\ * FEI Company, Application Laboratory, P.O. Box 80066, 5600 KA Eindhoven, The Netherlands
}

The small dimensions and complex structures encountered in nano-technology and semiconductor devices result in new challenges for characterization techniques and an increasing need for threedimensional structural information. Electron tomography is a widely recognized method (in lifescience) to obtain high-resolution three-dimensional images by electron microscopy [1]: A sample is tilted around a single axis over a wide angular range $\left( \pm 60-70^{\circ}\right)$ with electron micrographs obtained at small angular tilt increments [2]. Subsequently, the images of the tilt series are aligned and a reconstruction of the three-dimensional volume is calculated based on an interpretation of the TEM images in terms of mass-thickness (Figure 1).

The methodology of electron tomography can also be extended towards materials science, nanotechnology, and IC applications. However, due to diffraction contrast from these (crystalline) materials, the classical approach using TEM bright field will be limited, as the contrast will strongly change over the tilt range. This results in a (significant) noise in the reconstructed image volume, which considerably limits an unambiguous interpretation of the results.

We are currently exploring the use of other electron microscopy techniques, such as ADF-STEM, HAADF-STEM, energy-filtered TEM, and hollow-cone TEM as alternative tomography methods for 3-D visualization of crystalline inorganic materials. In addition to reducing diffraction contrast, these electron microscopy techniques further need to result in 2D images that directly reflect the projected elemental- or mass-thickness in order to yield meaningful electron tomography reconstructions. Our evaluation of the above-mentioned methods indicates, that HAADF-STEM imaging results in accurate high-resolution electron tomography reconstructions for a wide range of applications such as life science [3], materials science [4], nano-technology, and the semiconductor devices (Figure 2). To mention one of the examples for HAADF-STEM tomography, Figure 2 shows an FIB prepared IC device, where the 3D reconstruction reveals defects that are not visible or cannot be localized in a simple 2D projection. Further details on the electron microscopy methods for tomography will be presented during the talk together with applications examples.

\section{References}

[1] J. Frank, Three-Dimensional Electron Microscopy of Macromolecular Assemblies, Academic Press, San Diego, 1996.

[2] Visit the FEI booth for a demonstration of the Tecnai ${ }^{\mathrm{TM}}$ G2 Sphera for fast and accurate automated TEM tomography.

[3] U. Ziese, C. Kübel, A.J. Verkleij, A.J. Koster, J. Struct. Biology, submitted.

[4] P. Midgley et al., Chem. Commun. (2001) 907. 

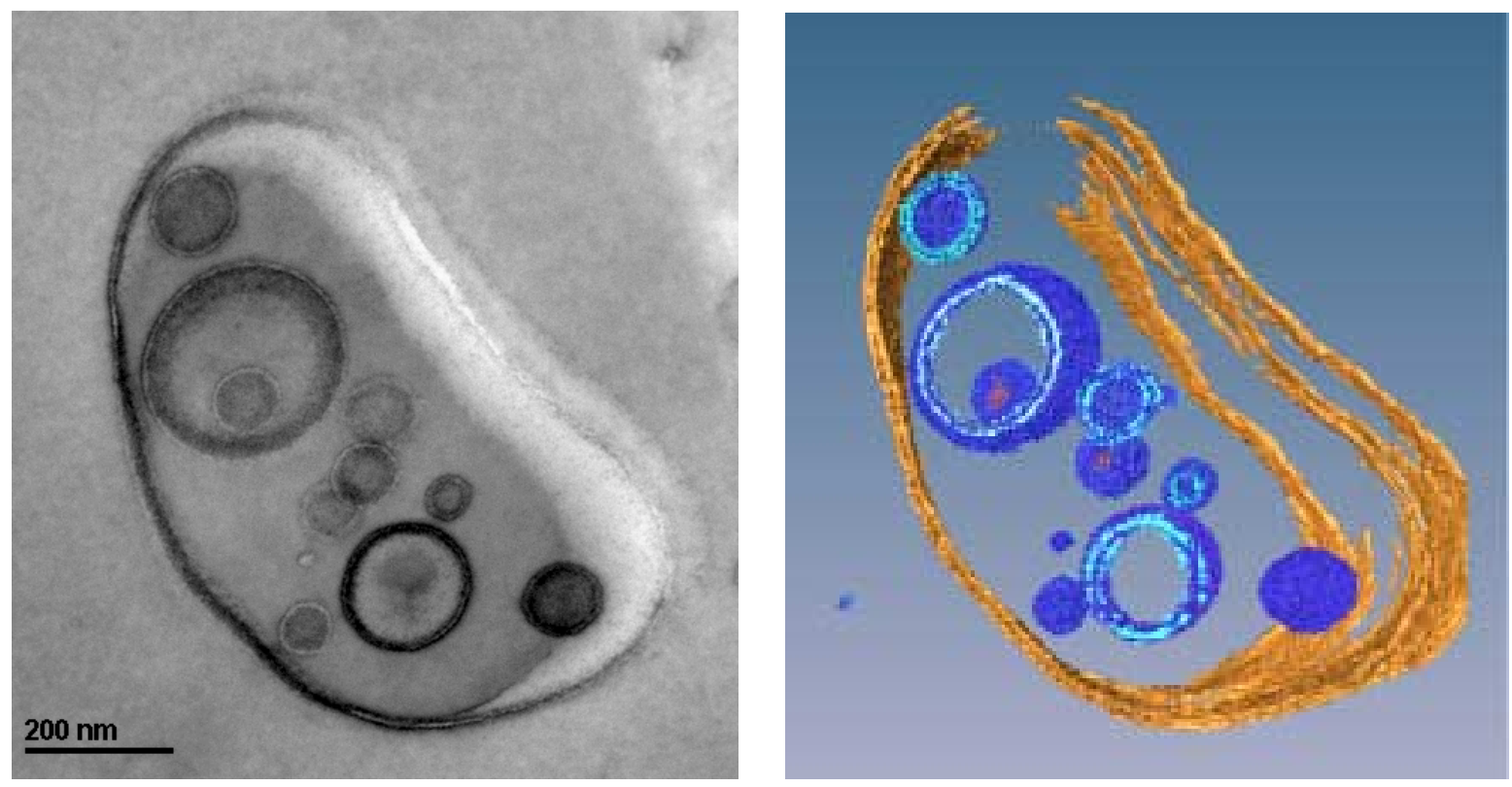

FIG 1. Electron tomography example showing a reference image at $0^{\circ}$ tilt and the reconstructed 3D volume of a $150 \mathrm{~nm}$ thick epoxy section with stained block-copolymer vesicles. The bilayer was resolved in 3D with a feature width of $3 \mathrm{~nm}$.
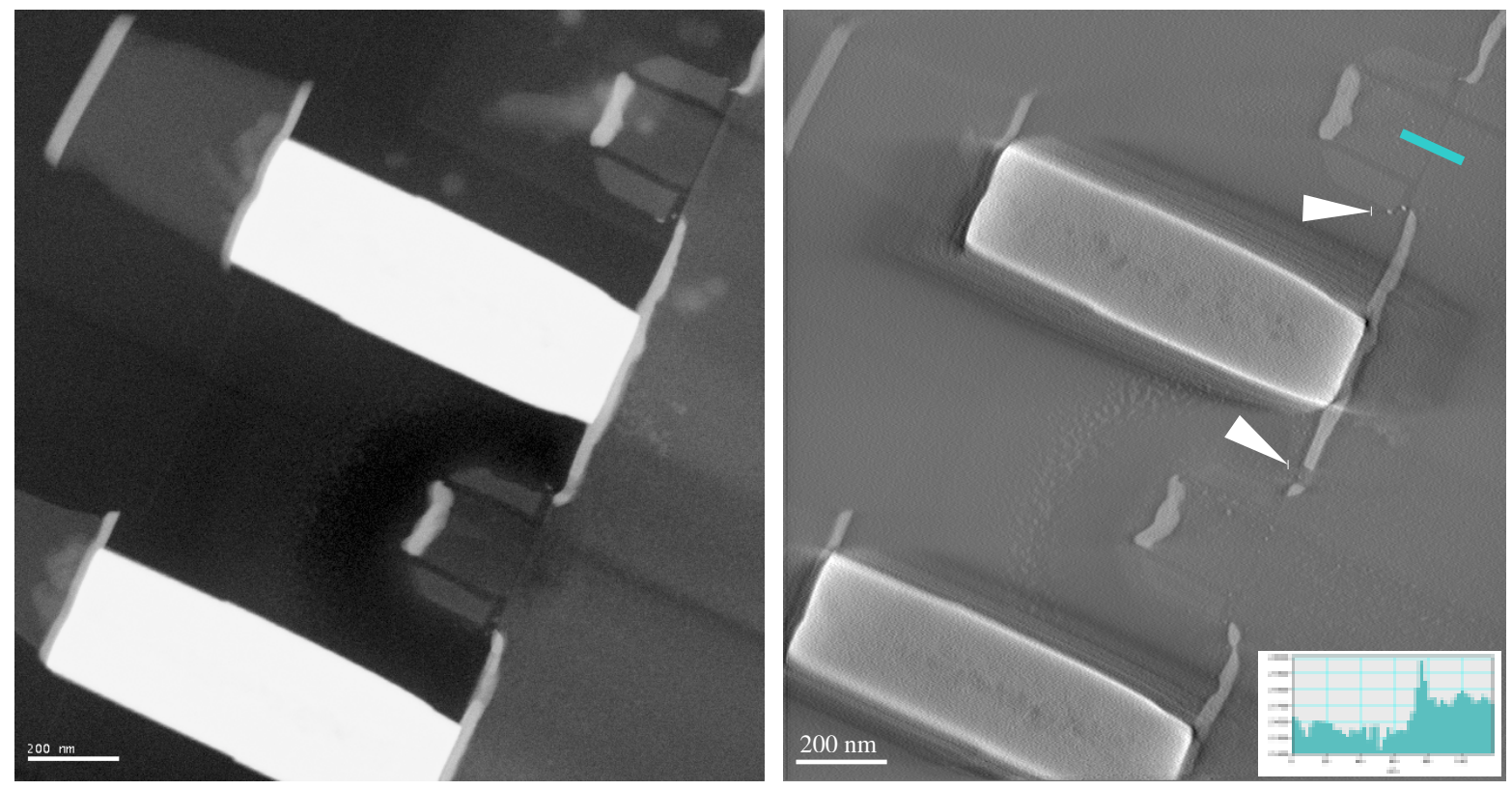

FIG 2. HAADF-STEM reference image at $0^{\circ}$ tilt and a slice of the reconstruction of a $150 \mathrm{~nm}$ thick semiconductor section (transistor, tungsten via, aluminum connect) prepared by FIB (the inset shows a line profile of the gate oxide). In the reconstructed volume, various defects and the gate oxide are visible in $3 \mathrm{D}$ as well as variations of the barrier layers and holes in the via. 